\title{
Dielectric Elastomer Spring Roll Actuators for a Portable Force Feedback Device
}

\author{
Rui Zhang* \\ Swiss Federal Institute of Technology (ETH) \\ Center of Product Development \\ Tannenstrasse 3, 8092 Zurich, Switzerland \\ Patrick Lochmatter \\ Swiss Federal Laboratories \\ for Materials Testing and Research (Empa) \\ Laboratory for Materials and Engineering \\ Ueberlandstrasse 129 \\ 8600 Duebendorf, Switzerland
}

\author{
Andreas Kunz ${ }^{\dagger}$ \\ Swiss Federal Institute of Technology (ETH) \\ Institute of Machine Tools and Manufacturing \\ Tannenstrasse 3, 8092 Zurich, Switzerland \\ Gabor Kovacs $\S$ \\ Swiss Federal Laboratories \\ for Materials Testing and Research (Empa) \\ Laboratory for Materials and Engineering \\ Ueberlandstrasse 129 \\ 8600 Duebendorf, Switzerland
}

\begin{abstract}
In this paper, we present a new portable force feedback device for surgery simulations. Dielectric elastomer spring roll linear actuators for this device were manufactured, and characterized via passive tensile tests and active isometric tests. The actuators exhibited a maximum force of $7.2 \mathrm{~N}$, and a maximum elongation of $31 \%$. Due to the high driving voltage, electrical safety issues were also considered. The results showed that sufficient electrical safety can be provided to the user. Two prototypes were built, which practically showed functionalities of the actuator and the proposed force feedback concept with actuators connected between the fingers.
\end{abstract}

CR Categories: H.5.2 [Information Interfaces and Presentation]: User Interfaces-Haptic I/O; H.1.2 [Models and Principles]: User/Machine Systems-Human Factors

Keywords: force feedback, actuator, dielectric elastomer, electrical safety

\section{INTRODUCTION}

The design of a force feedback device is always an applicationoriented task due to the complex haptic sensation of human beings and the limitation of the nowadays actuation technologies. This holds also true for the development of a force feedback device for open surgery simulations.

Our study shows that surgeons use their hands $22-26 \%$ of the whole operation time to touch the body of a patient, to define the operation area, or to find, hold and to palpate the organ [22]. From the organ palpation, surgeons receive precise haptic information of the organ such as texture, temperature, humidity, stiffness, etc. The fingertips of the index, the middle finger, and the thumb are mainly involved in this process. According to the classification of grasping geometries [7], this action belongs to the precision grasping.

Force feedback devices used for grasping simulations can generally be classified into ground-based and body-based types according to the location of the device. The former is fixed to its environment such as desk, ceiling, wall, or floor, while the latter has its ground on the user's body.

\footnotetext{
*e-mail:rui.zhang@imes.mavt.ethz.ch

†e-mail:kunz@iwf.mavt.ethz.ch

†e-mail:patrick.lochmatter@empa.ch

$\S$ e-mail:gabor.kovacs@empa.ch
}

Symposium on Haptic Interfaces for

Virtual Environment and Teleoperator Systems 2006

March 25 - 26, Alexandria, Virginia, USA

1-4244-0226-3/06/\$20.00 O2006 IEEE
Some of the ground-based devices are based on stylus apparatus such as PHANTOM [21] or Delta [16]. In order to study visual recalibration aided by haptic cues, Atkins et al [3] have used two PHANTOMs, allowing a user to grasp virtual objects with his thumb and index finger.

Barbagli et al [4] have attached additional force reflecting grippers to two PHANTOMs, which enable multi-finger, dual-hand explorations of virtual objects.

The advent of these ground-based devices enable users to intuitively grasp and manipulate virtual objects. However, implicit friction and backlash from the series design, limited workspace, absence of portability, and high cost of two complete systems are their drawbacks.

Body-based devices are portable and provide more workspace to users in virtual environments (VE). These devices provide forces onto the fingertips, either from the dorsal side, or from the palmar side of the hand.

Stergiopoulos et al [13] have developed a two-finger hand exoskeleton with 2 active degrees of freedom (DOF). The device is driven by DC motors that are located at the metacarpophalangeal $(\mathrm{MCP})$ joints. Forces are transmitted onto each fingertip via a cablepulley mechanism.

Instead of positioning the actuators on the hand, CyberGrasp [18] has 5 electric actuators located remotely from the hand. Their forces are transmitted onto the fingertips via a cable-pulley system as well.

Using ultrasonic actuators, Choi et al [6] have designed a hand exoskeleton with a 4-bar mechanism transmitting the forces onto the fingertips.

Bouzit et al [5] have developed Rutgers Master II with four pneumatic actuators located between the palm and the fingers (except for the little finger). This structure requires no force transmission mechanism (kinematic-free), and provides forces directly onto fingertips, where contact forces are necessary.

Compared to ground-based devices, portable devices enable users to interact with virtual objects in a more intuitive way. However, the portability is achieved at the sacrifice of their ergonomics.

Force transmission mechanisms can be found in both types of devices. Such mechanisms result in bulky, and mechanically complex systems with high inertia and high internal frictions. It further induces an obscuring of the effective feedback force (actuator force/system friction). The adjustment of such mechanisms to fit different sizes of hands is time-consuming and may cause errors in the position sensing. Moreover, the attachments of these transmission structures on the hand introduce unwanted forces, which may strongly disturb the user from concentrating on a task.

In our previous study [9] and [24], we presented a conceptual force feedback glove with integrated tendon-like actuators on the 
finger's dorsal side. The tendon-like actuator provide forces on the fingertips by pulling them backwards. No force transmission structure is necessary. An elementary dielectric elastomer (DE) actuator for this concept (active area: $48 \times 30 \times 0.1 \mathrm{~mm}^{3}$ ) exhibited a displacement of $7.5 \mathrm{~mm}$ and a contractile force of $0.7 \mathrm{~N}$ under a pre-stretching force of $2 \mathrm{~N}$.

The challenge for such planar DE actuators is the design of a housing, which should be able to hold biaxial pre-strains in the film (VHB 4910 3M) while allowing one degree of freedom for the active deformation. We tried both joint-free housings and mechanical slides with spiral springs. The former was not able to hold the prestrains completely. The latter strongly reduced the ratio of the active material's mass to the housing's mass. Due to those difficulties in the actuator manufacturing and the required external pre-stretching force, the application of this tendon-like actuator for the proposed force feedback glove was limited.

In this study, we present a DE spring roll actuator, which has been improved concerning the actuator construction, manufacturing process, actuator performance, and its suitability for a new portable force feedback device. The final goal of this study is to build a kinematic-free, lightweight, and portable force feedback device for, but not limited to surgery simulations.

\section{Concept Study of Glove-like Force Feedback DEVICES}

In order to determine the functions of a portable force feedback device, we need to look at the essentials of human-object grasping.

In precision grasping, the index finger, the middle finger, and the thumb are involved with the contact forces concentrating on the fingertips [7]. The forces from the object act against the finger's flexion, which is generated by the muscles and tendons in the hand and the forearm (see Figure 1(a)). Here, we assume that the contact force is normal to the contact surface.

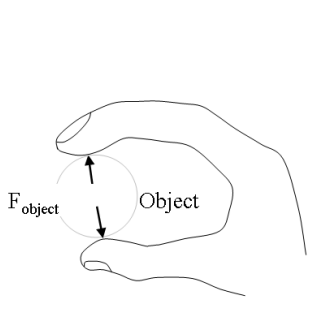

(a)

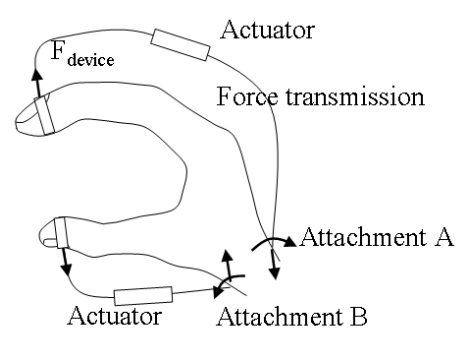

(b)
Figure 1: (a) External resulting forces from the object act against the finger flexion in precision grasping and (b) the contact model of the finger-device grasping.

To simulate an object grasping in VE, a device must be able to provide resulting external forces on the contact areas. As shown in Figure 1(b), we take a portable device, which is grounded on the wrist for example. Local actuators with force transmission mechanisms are able to provide the required force.

However, there exist constraint forces from the device acting on the wrist via the attachment A and B. As we mentioned in Section 1 , these unnecessary forces consequently reduce the fidelity of the force feedback, since they don't exist in a real object grasping. Therefore, it is important to moderate the effects of these constraint forces on the user by properly positioning the actuators, properly selecting and attaching a transmission system to the hand.

\subsection{Requirements}

In this study, we've defined two major functional capabilities for designing a transparent [4] force feedback device.

(i) Force feedback capability: The device should be able to generate forces at given contact points, when an interaction between a virtual hand and a virtual object occurs. Here we defined one actuator at one contact point as one active DOF. For the aforementioned precision grasping, four active DOF are required with each for the fingertip of the index, the middle, the ring finger, and the thumb.

(ii) Backdrivablility: The device should have low intrinsic mechanical impedance, so it can passively follow an imposed motion from the finger. Extending this definition, a device is also considered to be backdrivable if it can actively follow the imposed motion. Thus, the user can move their fingers freely without perceiving the existence of the device. The DOF of the fingers' motions is defined here as passive DOF. 16 passive DOF are considered to be enough for the precision grasping simulation (four for each finger, except for the little finger).

Detailed functional requirements such as force, elongation, bandwidth, etc. were given in [24]. In addition, ergonomics such as weight, volume, location, and kind of the attachment onto the body were considered.

\subsection{Concepts for a portable force feedback device in three cat- egories}

For portable devices, the fixation of the devices is limited to the hand including the wrist. According to the locations of the actuators, we have classified our concepts into three categories: actuators on the dorsal side, on the palmar side, and actuators around the fingers. The concepts is briefly analyzed concerning their mechanics (distribution of the feedback force and their counter forces from the attachments, force direction, etc.), as well as their ergonomic factors (design space, weight, volume, inertia, friction, etc.).

\subsubsection{Actuators on the dorsal side}

Most of the existing portable devices belong to this group, such as the above mentioned CyberGrasp, the 2-finger hand exoskeleton, and the ultrasonic based hand exoskeleton. The devices provide forces onto the fingertips by pulling them backwards, either having the actuators integrated in the exoskeleton or remotely located. Additional force transmission mechanisms are required for introducing the actuator forces normal to the fingertips.

To avoid any force transmission, we can attach tendon-like DE actuators on the finger's back [24], or integrate such actuators in a protagonist-antagonist mechanism over the fingers. A lightweight and concise structure can therefore be reached. However, the force acts more along the finger axis than normal to the fingertip. Obviously, bulky force transmission mechanism and a realistic force become a tricky trade-off in this category.

One advantage of this group is their large design space on the dorsal side. Depending on their force transmissions, they could be kinematic-free, and provide wide area feedbacks [8]. The actuators can be individually controlled, which allows rich simulation metaphors.

\subsubsection{Actuators on the palmar side}

Instead of pulling the fingers backwards, the actuators in this category provide forces onto the fingertips by pushing against them. They can be attached between the thumb and other fingers, between 
the finger phalanxes, between the fingers and the palm (such as Rutger Master II), or between the fingers and the wrist, respectively.

Compared with the category actuators on the dorsal side, this group also allows individual control of the actuators and wide area force feedback, but it has less design space. No force transmission mechanism is required, and thus, it allows a light-weight, and concise device with low friction, low inertia, and zero backlash.

Furthermore, some concepts take great use of the counter grounding force and transfer it into the useful feedback forces. Taking the Rutgers Master II for example, it can well simulate powerful grasping, where the fingers and the palm are involved. There is no unrealistic constraint force (as from force transmission mechanisms) that disturbs the users.

One drawback of these systems is the limited simulation metaphors (either touch or grasping, but not both).

This group contains instability as well. Opposite to the actuators on the dorsal side, the actuator and its attachments are under compressive load. Due to the softness of the fingers and their perception constraints, an ideally rigid connection between fingers and actuators is not possible. Furthermore, the compressive force on the actuator is limited by its buckling load. These factors may seriously deteriorate the force feedback fidelity.

\subsubsection{Actuators around the fingers}

In this group the actuators are directly attached around the finger phalanxes. Such as ring-like actuators or sphincter-like actuators, the actuators provide forces to the fingertips by radially squeezing the fingers.

Due to the limited ab-/adduction angles of the fingers, there is little design space. Moreover, the resulting external force from the sphincter-like actuator on the fingertips is almost zero. According to our discussion on the contact model (see Figure 1), we doubted if this category should be taken into account. Therefore, we made initial psychophysical tests in order to find out if the sphincter-like actuators would provide realistic grasping forces to the user.

A textile band was attached around the subject's index fingertip. Two ends of this band were held by the examiner. By contracting these two ends, the examiner can manually "switch on" (i.e. contracting) the part around the finger, and thus squeezing forces were applied around the fingertip.

Eight subjects were asked to fulfill the tests. They were not informed about the content of the test before. Each subject was asked to follow two phases: Phase I: forces were applied around the fingertip without any visual cue to the user, and Phase II: forces were applied around the fingertips, while visual cues were given to the user.

In Phase I, no subject could describe exactly what actually happened on the fingertip. However, in Phase II, seven of the total eight subjects felt a grasping force on the fingertip. Guided by the visual cues, the subjects could concentrate on the task of "grasping a glass", and were not disturbed by forces at the finger's dorsal and lateral side.

\subsection{Results of the concept study}

22 concepts in three categories have been established. Structure mechanics of each concept was studied in order to theoretically estimate the feedback resp. counter force distributions, and to specify the actuator requirements. 10 concepts with their feasibilities for DE actuators were selected, and then evaluated using the pair comparison method [10], out of which 4 solutions have been further studied.

The first rank, and the selected concept in this paper as well, was in the category actuator between fingers. Four actuators connect directly the thumb with other fingers. Such a solution has almost the same grasping geometry as human beings naturally have. The working principle of the corresponding DE actuators is introduced in Section 3.

\section{Spring Roll DE ACtuators}

Basically, a DE actuator is a compliant capacitor. A thin elastomeric film is sandwiched between two compliant electrodes. Under activation with a high DC voltage $(k V)$, the electrostatic pressure squeezes the elastomer film in thickness, and thus the incompressible film expands in all planar directions.

A DE spring roll actuator consists of biaxially pre-stretched, electrode-coated elastomer films, which are wrapped around an elastic core. The geometry of the core can be varied according to a given application. By utilizing such a compact structure, the energy density is significantly enhanced when compared to the foregoing planar DE actuator. Pei et al [12] [11] have developed multi-DOF DE spring roll linear or bending actuators for multi-leg robots. Having low buckling strength, the spring roll actuator provides limited compressive forces as needed for the selected concept actuator between the fingers.

\subsection{Manufacturing of the DE actuator}

For our application, we enhanced the core by adding a telescopic structure inside the spring. The DE spring roll linear actuators (see Figure 2) were produced at Empa Dubendorf [15] based on a halfautomated manufacturing process.

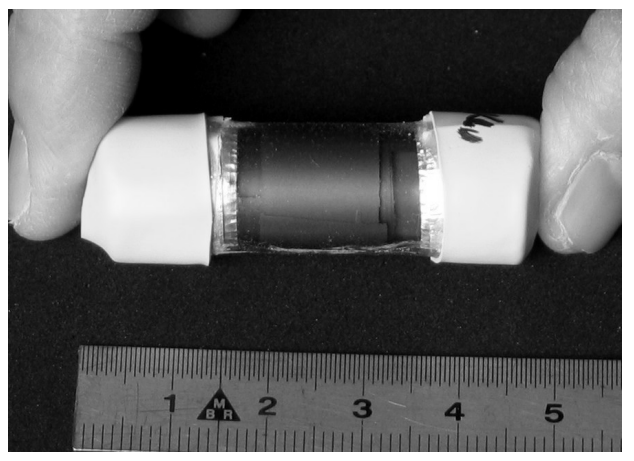

Figure 2: The DE spring roll linear actuator made at Empa Dubendorf.

Major steps of the actuator manufacturing are as follows: automated biaxial pre-stretching of the elastomer film (VHB 4910 $3 \mathrm{M})$, manual coating the films with compliant electrodes, manual wrapping of the pre-stretched film around the fully compressed telescope-spring core (see Figure 3 Assembly), and lateral fixation of the film on the core.

\subsection{Working principle of the DE spring roll linear actuator}

Basically, the DE spring roll actuator is an active spring with adaptive stiffness. Figure 3 schematically shows the working principle of the actuator for a force feedback device with actuators between the fingers. In the force-displacement diagram (see Figure 3), we assume that the employed VHB 4910 elastomer film is incompressible, isotropic, and has quasi-linear behavior under the given prestretch condition.

(i) Passive equilibrium: The fully compressed spring and the prestretched elastomer film come to a longitudinal force equilibrium, and thus a free-standing linear actuator is achieved.

As shown in Figure 4, the elastomer film is pre-loaded in all three dimensions in this equilibrium: in planar directions from 


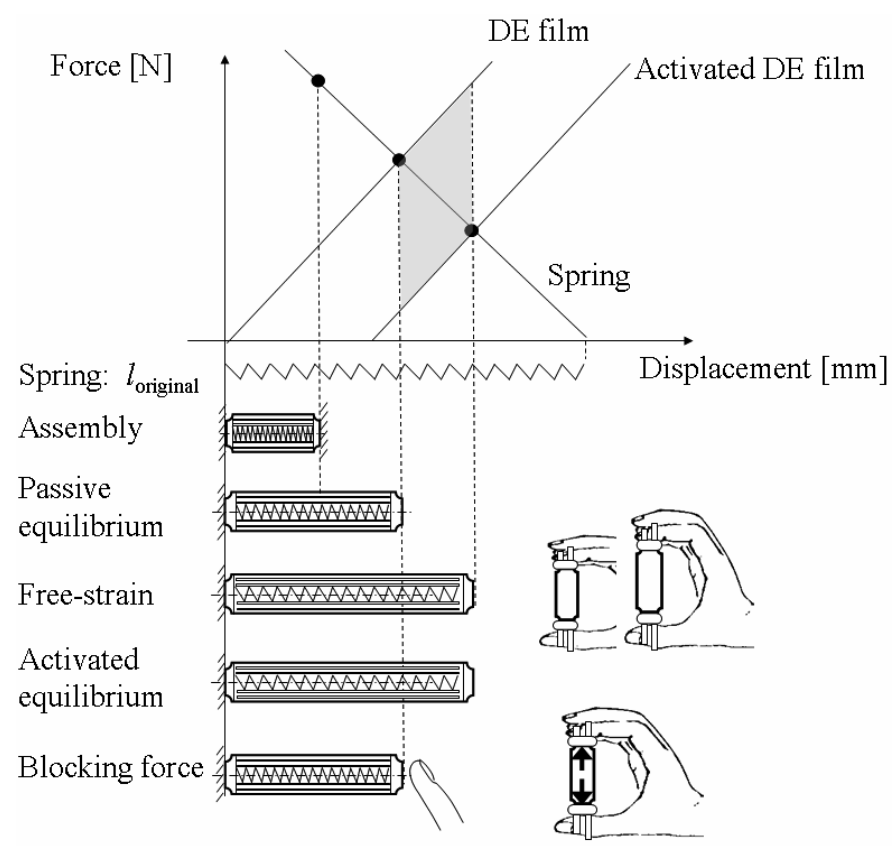

Figure 3: The working principle of DE spring roll linear actuators for the proposed force feedback device.

the pre-stretching, and in the film's thickness direction from the wrapping of the film around the cylindric core. An electrical voltage acting in the film's thickness direction brings electromechanical pressure in this direction. This force changes the force equilibrium state, and therefore the actuator can be controlled via the electrical voltage.
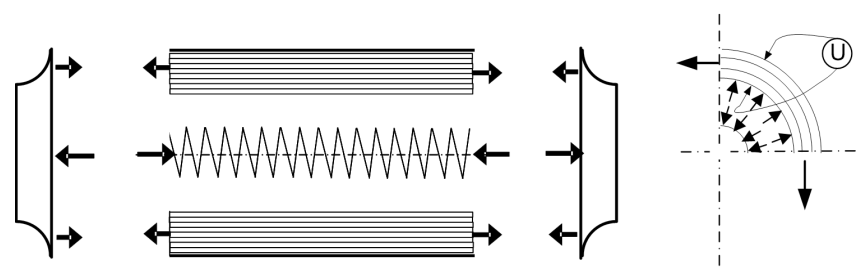

Figure 4: Force equilibrium of the free-standing actuator and the electromechanical coupling.

(ii) Free-strain under isotonic activation: If a high voltage is applied to the actuator, electrostatic forces act in the film's thickness. This results in a longitudinal elongation of the film. Consequently, the spring extends until the film and the spring get to a new force equilibrium called activated equilibrium. This motion is reversible when turning off the voltage. The free-strain enables the device to actively follow a finger's motion (backdrivability).

(iii) Blocking force under isometric activation: In the passive equilibrium, if the actuator's length is kept constant, and a voltage is applied to the actuator, the tensile force from the film decreases, and the spring tends to elongate. The resulting force can act against an external constraint (e.g. the finger) and is called blocking force of the actuator.

The free-strain and blocking force are two major characteristics for such an actuator. By varying the applied activation voltage and the actuator's boundary condition, all states within the shaded area in Figure 3 can be actively achieved.

\subsection{Characterization of the DE spring roll linear actuator}

In order to determine performances of the actuator, passive uniaxial tensile tests and isometric tests under activation were performed.

\subsubsection{Measurement setup}

As shown in Figure 5, we attached one side of the actuator to the grounded-frame, and the other side to a force sensor (HBM U2B [17]), which is mounted on a pneumatic cylinder (BRAMATI [15]). The displacement of the actuator was measured by the laser sensor (OADM20I6460/S1 4F, [14]). The measurement signals are controlled by LabView via an optoelectronic coupler [20] and a data acquisition card (BNC 2090 [19]).

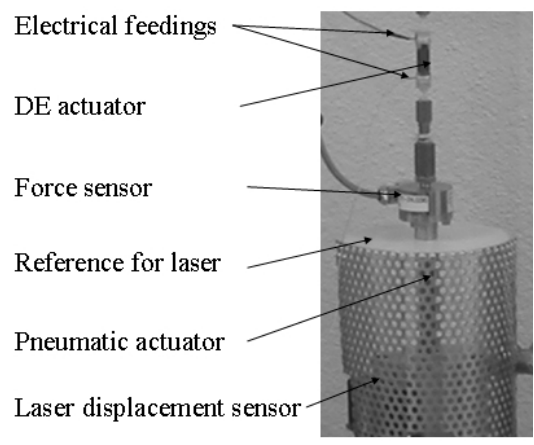

Figure 5: Setup of the tensile and isometric tests.

\subsubsection{Measurements}

In the passive uniaxial tensile tests, the actuator was stretched by the pneumatic cylinder from its free-standing length to $10 \mathrm{~mm}$ with an elongation rate of $2 \mathrm{~mm} / \mathrm{min}$. The tensile force of the actuator was measured.

During the isometric test under activation, two control signals were given by LabView: a DC activation voltage (provided by PS350, Stanford Research Systems) for the actuator, and a displacement control for the pneumatic cylinder. First, the actuator was kept in its passive equilibrium length. To avoid electrical breakdown caused by an overshooting voltage from a square activation signal, a DC activation voltage was linearly increased from 0 to $3.5 \mathrm{kV}$ within $90 s$ and applied to the actuator. The compressive blocking force from the actuator was measured.

Second, the actuator was stretched to $1 \mathrm{~mm}$ with an elongation rate of $2 \mathrm{~mm} / \mathrm{min}$. While keeping this distance and applying again the voltage up to $3.5 \mathrm{kV}$, the longitudinal force was measured. This step was repeated by stretching the actuator each time $1 \mathrm{~mm}$ more, until a maximum displacement of $10 \mathrm{~mm}$ was reached.

\subsubsection{Results}

We tested 30 actuators in different groups with slight differences in constructions and aging (due to the viscosity of the elastomer film). All results show a quasi-linear force-displacement behavior of the actuators. As shown in Figure 6, the measured force decreased as the linearly increasing voltage was applied for all initial pre-strains in the range of $0-10 \mathrm{~mm}$. At the original length of the actuator, this force varied in negative range (thrust). As we increased the displacement of the actuator, the force varied from negative area 


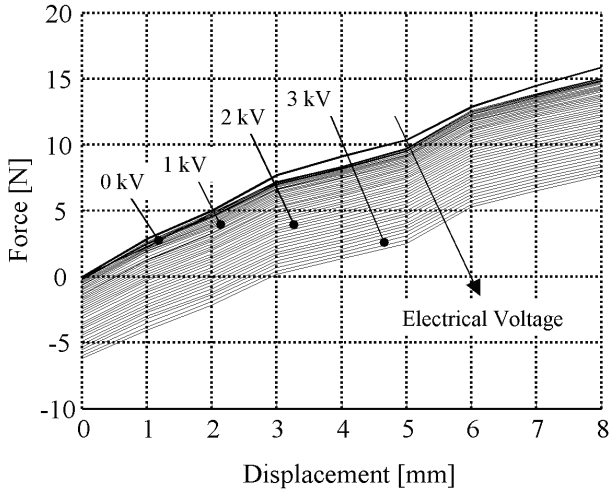

Figure 6: Force-displacement-voltage behavior of the DE actuator under isometric conditions.

(thrust) to positive area (tensile). From a certain displacement on, the force varied only in the tensile area.

For the application in the presented force feedback concept, both compressive and tensile forces can be effectively used depending on the required simulation (where one, or bidirectional force feedback is necessary). Figure 6 shows that a maximum compressive blocking force of $6 \mathrm{~N}$ was found at its passive equilibrium length of $45 \mathrm{~mm}$. This blocking force decreased to $0 N$ as a displacement of $3 \mathrm{~mm}$ was reached. This displacement of $3 \mathrm{~mm}$ represented the maximum displacement of the actuator. We also observed that this

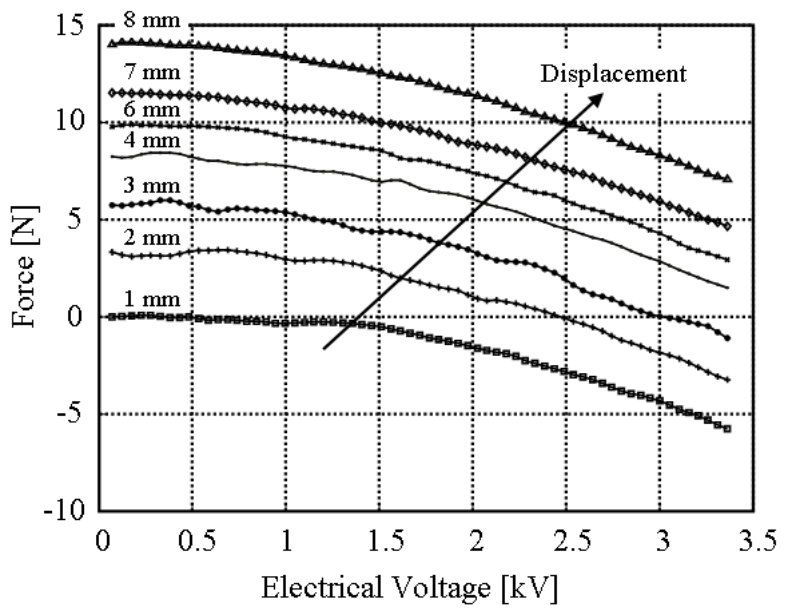

Figure 7: Force-voltage curve under isometric conditions.

force decreased non-linearly as the applied voltage was increased (Figure 7).

The maximum compressive blocking force of the 30 actuators, which can be effectively used for a force feedback device, ranged from 1.47 to $7.2 \mathrm{~N}$, while the maximum elongation varied from 1 to $5 \mathrm{~mm}$. These wide variances originated mainly from the deficiencies in the actuators' constructions, and in the manual manufacturing process for the actuator. The response time of the actuator could not be clarified in this experiment since a ramp voltage signal was used and the voltage applied to the actuator was not measured. As expected from the viscoelastic material behavior of the acrylic film, all results exhibited a strong time-dependency, which brings difficulties in the actuator control.

Table 1 shows technical data of the DE spring roll linear actuator.
Table 1: Technical data of the DE spring roll linear actuator.

\begin{tabular}{ll} 
Item & Specification \\
\hline Material & VHB 4910 3M \\
Dimension & $\emptyset=12 \mathrm{~mm}, \mathrm{~L}=45 \mathrm{~mm}$ \\
Layers & about 30-40 \\
Weight & $8 \mathrm{~g}$ \\
Activation voltage & $3.5 \mathrm{kV}$ \\
Max. thrust force & $7.2 \mathrm{~N}$ \\
Max. stroke & $5 \mathrm{~mm}$ \\
Manufacturing time & $67 \mathrm{~min}$ \\
Active area & $16 \times 1840 \mathrm{~mm}^{2}$
\end{tabular}

\section{Electrical Safety IsSues}

Sufficient electrical safety should be provided to the human operator since the $\mathrm{DE}$ actuator is driven by a high voltage. However, there is few publication focusing on the electrical properties of the DE actuator, and thus on how the electrical safety of the user can be guaranteed.

In order to characterize the possible endangerments to the user, a complete setup of the human operator, the actuator, cable, and power supply is represented by the electrical model shown in Figure 8. Possible failures of the system and thus endangerments to the

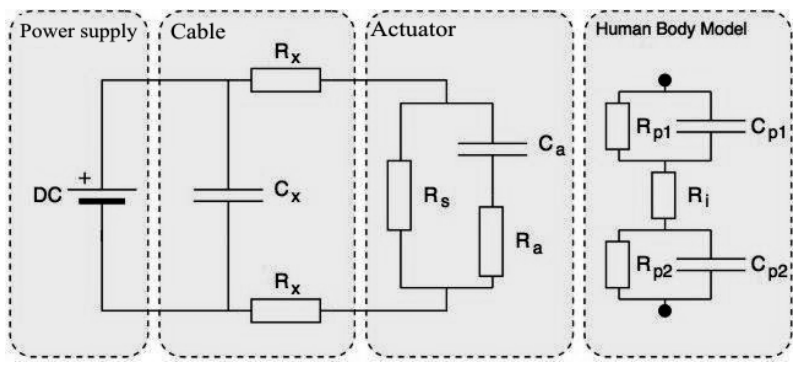

Figure 8: Electrical model of the overall system [2] [23].

user could occur mainly in two ways: the actuator, i.e. the capacitor would be discharged through the human body with (all blocks in Figure 8 are interconnected) or without being connected to any external power supply (only the two most right blocks of Figure 8 are connected). In both cases, the power supply could be a portable one or stationary one.

To determine the maximum discharge current and its time constant in the above failure cases, we measured the electrical properties of the actuators $\left(R_{a}, R_{s}\right.$, and $\left.C_{a}\right)$, defined the human body's electrical resistance concerning the discharge path, and the parameters (such as driving voltage, cable length etc.) in the circuit of the test setup (shown in Table 2). If the actuator would be discharged through the human body without being connected to any power supply, a maximum effective current of $22 \mathrm{~mA}$, an impulse duration of $7 \mathrm{~ms}$, and a charge of $0.125 \mathrm{mC}$ were calculated by the Simulink simulation at a given driving voltage of $5 \mathrm{kV}$. The wire's length between the actuator and the power supply was assumed to be 0.1 $m$ for a mobile supply, or $2 m$ for a stationary supply. According to IEC 60479-2 [1], this discharge through human body may cause pain at the contact area, but is not dangerous to the user. Nevertheless, the actuator was designed in such a way that the user would not be disturbed even in case of a failure. This was achieved, for example, by insulating the whole actuator, by connecting the outer electrode to a low potential, and by electronically limiting the maximum output current of the high voltage supply to $25 \mathrm{~mA}$. 
Table 2: Key parameters for the Simulink simulation.

\begin{tabular}{cccc}
\multicolumn{4}{c}{ Actuator parameter } \\
\hline$C_{a}[n F]$ & min & avg. & $\max$ \\
$R_{a}[k \Omega]$ & 2.5 & 13 & 25 \\
$R_{S}[k \Omega]$ & 90 & 300 & 700 \\
\multicolumn{5}{c}{ Human being parameter } \\
\hline & min & avg. & max \\
$R_{i}[\Omega]$ & (Hand-hand) & & (Hand-foot) \\
& 500 & - & 1000
\end{tabular}

\section{PRototypes}

In order to provide sufficient electrical safety to the user, the DE actuator was mounted inside a transparent plexiglas cylinder as shown in Figure 9(a). Touching the holders on both sides the user can feel the force and stroke generated by the actuator.

As shown in Figure 9(b), a prototype for a force feedback device was built as well. Three DE actuators were attached between the thumb and the index, the middle, and the ring finger via spherical joints. This system gives four DOF to each finger (flexion of the phalangeal joints, ab-/adduction of the metacarpophalangeal joint).

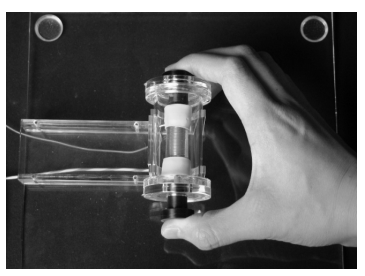

(a)

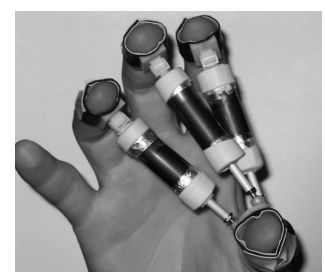

(b)
Figure 9: Prototype for the actuator (a) and for the force feedback device (b).

\section{Conclusions And Discussions}

In this paper, we theoretically compared different concepts for portable force feedback devices, and presented a new concept with actuators between the fingers. The prototype based on the DE actuators provided us a promising feedback force.

The device has a total weight of $38 \mathrm{~g}$, which is a progress compared to the Rutger Master II (80 $g$ ) and the CyberGrasp (340 $g$ ). However, the device's performance measurement (such as elongation and force), and psychophysical tests need to be done in order to prove the concept. Mechanisms amplifying the output displacement or force may be necessary so that diverse material properties (such as soft tissues) can be rendered.

Dielectric elastomer spring roll linear actuators were manufactured based on a half-automated manufacturing process. The actuator exhibited a maximum blocking force of $7.2 \mathrm{~N}$, and a maximum elongation of $31 \%$ according to the active length (11\% according to the total length). The high driving voltage does not endanger the user. Having the advantages of light weight $(8 g)$, no operation noise, compact and portable construction, such DE actuators are promising for many applications including robotic, automation and entertainment industry etc.

However, performances of the actuator such as efficiency, response time to a square voltage signal, working cycle, and time- dependency (concerning the loading rate in the active isometric tests) need to be further characterized and compared with conventional actuators. The actuator reliability will be improved by optimizing its mechanical and electrical design. To overcome the viscoelastic behavior of the actuator, either new dielectric films with less viscosity (e.g. silicone films [25]), or appropriate control methods have to be studied. Manual work in the manufacturing process for such miniaturized actuators resulted in poor efficiency and reproducibility. Therefore, a fully automated manufacturing process needs to be developed.

\section{ACKNOWLEDGEMENTS}

This study has been funded by the Swiss National Science Foundation, and been greatly supported by Swiss Federal Laboratories for Materials Testing and Research (Empa). Particularly, we would like to thank Mr. Lukas Kessler, Claudio Iseli, and Urs Hintermüller for their supports in actuator manufacturing, Mr. Hans-Jürg Weber for the electrical safety issues, Mr. Alfred Schmidlin, Florentin Gröli for the actuator characterization and the prototype construction, and Mr. Philipp Boehringer for the device design.

\section{REFERENCES}

[1] Effects of current passing throuth the human body. Part 2: Special Effects. Number IEC 60479-2. International Electrotechnical Commission (IEC), 2 edition, 1987.

[2] Effects of current on human beings and livestock. Part 1: General aspects. Number IEC 60479-1. International Electrotechnical Commission (IEC), 3 edition, 1994.

[3] J. E. Atkins, Robert A. Jacobs, and David C. Knill. Experiencedependent Visual Cue Recalibration based on Discrepancies between visual and Haptic Percepts. Vision Research, 43:2603-2613, 2003.

[4] F. Barbagli, K. Salisbury, and R. Devengenzo. Enabling Multi-finger, Multi-hand Virtualized Grasping. In Proceedings of IEEE International Conference of Robotics and Automation, volume 1, pages 12591263, Taipei, ICRA 2003.

[5] Mourad Bouzit, Grigore Burdea, and George et al Popescu. The Rutgers Master II-New Design Force Feedback Glove. IEEE/ASME Transactions on Mechatronics, 7(2):256-263, 2002.

[6] B.H. Choi and H.R. Choi. SKK Hand Master-Hand Exoskeleton Driven by Ultrasonic Motors. In Proceedings of the 2000 IEEE/RS International Conference on Intelligent Robots and Systems, pages 1131-1136, 2000.

[7] M. Cutkovsky and R. Howe. Human Grasp choice and Robotic Grasp Analysis. Springer Verlag, 1990.

[8] Andrea Mazzone and Andreas Kunz. Sketching the Future of the SmartMesh Wide Area Haptic Feedback Device by Introducing the Controlling Concept for such a Deformable Multi-loop Mechanism. In Proceedings of the World Haptics Conference, 2005.

[9] Andrea Mazzone, Rui Zhang, and Andreas Kunz. Novel Actuators for Haptic Displays based on Electroactive Polymers. In Virtual Reality Software and Technology Conference, pages 196-204, Osaka Japan, 2003.

[10] Markus Meier. Product Development. Script, Institute of Product Development, ETH Zurich, 2002.

[11] Q. Pei, Marcus Rosenthal, Scott Stanford, Harsha Prahlad, and Ron Pelrine. Multiple-degrees-of-freedom electroelastomer roll actuators. Smart Materials and Structures, 13:N86-N92, 2004.

[12] Qibing Pei, Ron Pelrine, Scott Stanford, Roy Kornbluh, and Marcus Rosenthal. Electroelastomer rolls and their application for biomimetic walking robots. Synthetic Metals, 135-136:129-131, April 2003.

[13] P. Stergiopoulos, Philippe Fuchs, and Claude Laurgeau. Design of a 2-Finger Hand Exoskeleton for VR Grasping Simulation. In Eurohaptics, Dublin, Ireland, 2003.

[14] www.baumerelectric.com.

[15] www.empa.ch.

[16] www.forcedimension.com. 
[17] www.hbm.com.

[18] www.immersion.com.

[19] www.ni.com

[20] www.relmatic.ch

[21] www.sensable.com.

[22] Nadja Waelchli and Andreas Kunz. Development of an Haptic Feedback Interface: Requirements from surgeons. Semester thesis, ETH Zurich, Februrary 2002.

[23] Hans-Juerg Weber, Mathias Meyer, Tobias Tomaschett, and Rui Zhang. Electrical safety issues of the dielectrical elastomer actuators for a force feedback glove. Semester thesis, High Voltage Laboratory, ETH Zurich, July 2005.

[24] Rui Zhang, Andrea Kunz, Kovacs Gabor, Michel Silvain, and Andrea Mazzone. Dielectric Elastomer Actuators for A Portable Force Feedback Device. In Proceedings of Eurohaptics 04, pages 300-307, Muenchen, Germany, 2004.

[25] Xuequn Zhang, Michael Wissler, B. Jaehne, R. Broennimann, and G. Kovacs. Effects of crosslinking, prestrain, and dielectric filler on the electromechanical response of a new silicone and comparison with acrylic elastomer. In Smart Structures and Materials 2004: Electroactive Polymer Actuators and Devices (EAPAD), volume 5385, pages 78-86, San Diego, CA, USA, 2004. 